\title{
REVIEW
}

\section{Embryo-mediated genome editing for accelerated genetic improvement of livestock}

\author{
Zachariah MCLEAN ${ }^{1,2}$, Björn OBACK ${ }^{1,3}$, Götz LAIBLE (ه) $)^{1,3}$ \\ 1 Reproduction, AgResearch, Ruakura Research Centre, Hamilton 3214, New Zealand \\ 2 School of Biological Sciences, University of Auckland, Auckland 1010, New Zealand \\ 3 School of Medical Sciences, University of Auckland, Auckland 1023, New Zealand
}

\begin{abstract}
Selecting beneficial DNA variants is the main goal of animal breeding. However, this process is inherently inefficient because each animal only carries a fraction of all desirable variants. Genome editing technology with its ability to directly introduce beneficial sequence variants offers new opportunities to modernize animal breeding by overcoming this biological limitation and accelerating genetic gains. To realize rapid genetic gain, precise edits need to be introduced into genomicallyselected embryos, which minimizes the genetic lag. However, embryo-mediated precision editing by homology-directed repair (HDR) mechanisms is currently an inefficient process that often produces mosaic embryos and greatly limits the numbers of available edited embryos. This review provides a summary of genome editing in bovine embryos and proposes an embryo-mediated accelerated breeding scheme that overcomes the present efficiency limitations of HDR editing in bovine embryos. It integrates embryo-based genomic selection with precise multi-editing and uses embryonic cloning with elite edited blastomeres or embryonic pluripotent stem cells to resolve mosaicism, enable multiplex editing and multiply rare elite genotypes. Such a breeding strategy would enable a more targeted, accelerated approach for livestock improvement that allows stacking of beneficial variants, even including novel traits from outside the breeding population, in the most recent elite genetic background, essentially within a single generation.
\end{abstract}

Keywords animal breeding, cattle, cloning, CRISPR/ Cas9, cytoplasmic injection, embryo, genome editing, germline chimaeras, HDR, livestock improvement, TALENs

Received October 31, 2019; accepted November 27, 2019

Correspondence: goetz.laible@agresearch.co.nz

\section{Introduction}

Existing natural variation in the gene pool of domesticated animals has been successfully used to improve livestock, such as cattle, through conventional breeding by artificial selection. Although this strategy has been proven highly successful, progress is limited to small incremental steps at each generation, typically amounting to annual genetic gains of $1 \%$ to $3 \%$, which become more substantial over longer time frames ${ }^{[1]}$.

However, conventional breeding is slow. As every breeding event generates new gene combinations, which cannot be directed or controlled, each animal only carries a fraction of all potentially desirable sequence variants. To accomplish genetic improvement, it is important to select the best animals that will become the parents for the next generation of improved animals. Genomic selection, using genome-wide single-nucleotide polymorphism (SNP) chip arrays or whole genome sequencing, provides a powerful means for the identification of animals with the most favorable genotypes that assemble the overall best combination of sequence variants in an individual animal ${ }^{[2]}$. This concept, first introduced in $2001^{[3]}$, has allowed unprecedented advances in commercial breeding in the past 15 years, including a doubling of dairy cattle improvement per generation compared to traditional selection. However, the potential for genetic improvement can be compounded by genetic linkages that result in the concurrent inheritance of desirable and non-desirable sequence variants. Depending on how tight the genetic linkages are, passenger mutations may not be easily removed by breeding into subsequent generations, even if their identity is known.

The development of programmable nucleases allows for the precise site-specific editing of livestock genomes ${ }^{[4]}$. With genome editing technology, it is now possible to uncouple genetic linkages and directly introduce individual beneficial variants, even from outside the natural 
breeding population, into elite genetic backgrounds. Further, it facilitates the assembly of multiple high-impact sequence variants in individual genomes which can be very difficult or impossible to achieve with conventional breeding strategies. Presently, genome editing targets are still limiting due to a low number of known causative sequence variants with relatively high impact on phenotypic production traits. Decreasing cost for whole genome sequencing is now allowing the sequencing of large numbers of animals which can greatly facilitate the finemapping and identification of causative variants ${ }^{[5]}$. Likewise, the inclusion of increasing numbers of causative sequence variants is also expected to increase the accuracy of genomic selection.

Genome editing, in combination with genomic selection, offers a novel opportunity that could change the scope and speed for improving efficiency of livestock production and completely overhaul current livestock breeding practices. For increased speed of genetic gain of large animals with long generation times, such as dairy cattle, it is paramount that selection and editing is undertaken as early as possible, using appropriately elite baseline genetics.

Here, we review the current approaches of embryomediated genome editing in cattle, outline its shortcomings, suggest solutions to overcome these issues and present a model for an improved breeding strategy. We propose to combine embryonic genomic selection, genome editing and multiplication into a breeding platform that captures embryo-derived germlines as clones or germline chimaeras. This generates a contemporary breeding strategy offering large scale dissemination and accelerated genetic gain that is superior to currently implemented procedures.

\section{Genome editing for livestock breeding}

Programmable nucleases have provided the molecular tools to efficiently introduce defined sequence variants into livestock genomes. This enables the direct introgression of beneficial traits essentially within a single generation, which offers scope for improvements of animal breeding by directed and fast genetic gain.

\subsection{Double strand break (DSB)-dependent editors}

At present, the toolbox contains three main technology platforms. Zinc finger nucleases (ZFNs) were the first available genome editors, combining a programmable DNA binding domain of multiple zinc finger modules with the catalytic domain of the restriction enzyme FokI ${ }^{[6]}$. They were soon followed by the development of an analogous protein-based editing platform, transcription activator-like effector nucleases (TALENs) where the FokI domain was linked to a modular DNA binding domain consisting of an array of repeat units whose intrinsic polymorphic sites determine DNA binding specificity ${ }^{[7]}$. Both platforms were protein-based, which required customization of the DNA binding domain for each editing target, limiting flexibility and making it expensive. This changed with the advent of the much simpler clustered regularly interspaced short palindromic repeat (CRISPR)/CRISPR-associated nuclease 9 (Cas9) system $^{[8]}$. Rather than entirely protein-based, it is an RNAguided nuclease system that uses a universal monomeric nuclease (Cas9) and a small programmable guide RNA (gRNA), which guides the nuclease to the editing site through sequence complementarity. The gRNAs can be readily designed for unique genome target sites through various online tools and economically synthesized by commercial suppliers. Given that the Cas9 nuclease can be universally used for any target site, it is now commercially available as mRNA or recombinant protein. This provides unprecedented flexibility and easy access to cost-effective genome editors for any desirable target site.

All site-specific nucleases function by introducing a DSB into the genomic DNA at the programmed target site. A DSB in the genome is typically repaired by the cellular repair machinery via the non-homologous end joining (NHEJ) repair process. NHEJ is an error-prone process. Due to endogenous nuclease activity at the DSB, the repair process often leads to an introduction of small insertions or deletions (indels) at the target site. However, there is no control over how the target site is edited by these indels, which makes this mechanism unsuitable for directed breeding applications. This is different when a pair of editors is introduced which can efficiently delete the fragment between the two $\mathrm{DSBs}^{[9]}$. In this case, the outcome can be more predictable and used to introduce a naturally occurring deletion that is associated with a beneficial trait.

Many of the sequence variants relevant for breeding can be conversions, deletions or insertions of single base pairs. Their introduction requires full control over genome editing outcomes, which can be achieved through an HDR mechanism. The site-specific DSB generated by a genome editor can trigger HDR. In the presence of a homologous repair template provided in trans, the cellular repair machinery can then repair the DSB according to the repair template. This can be exploited for the introduction of defined mutations, such as a single base pair change, by supplying a repair template that specifies the intended sequence changes ${ }^{[10]}$.

\subsection{DSB-independent editors}

Although limited to the change of specific base pairs, a new concept for the introduction of precise changes was developed with base editing. This no longer requires the introduction of a DSB and its subsequent repair. Rather, it 
is based on a Cas9 with either one or both of its endonuclease domains inactivated, rendering it a nickase, able to only introduce a nick at the target site, or removing any endonuclease activity. These mutant Cas9 enzymes retain uncompromised target site binding and when fused to a deaminase, enable the direct conversion of a $\mathrm{C}-\mathrm{G}$ base pair into a T-A base pair and an A-T base pair into G-C base pair ${ }^{[11]}$. The limitation of base editors for conversion of only a subset of single base pair combinations might already be resolved by the development of a new innovative tool with great potential for precise genome editing ${ }^{[12]}$. For this novel editor, a Cas9 nickase was fused to a reverse transcriptase. Like Cas9, it is programmed by a single RNA molecule but has additional functional components, compared to a conventional gRNA. The RNA molecule for the new editor combines the function of the gRNA to guide the nickase to the target site with a primer and template for reverse transcription. When encoded in the RNA template section of the RNA molecule, intended edits, including indels and all 12 types of point mutations, can be efficiently introduced into the genome without the need for a DBS or other exogenous DNA molecules as repair template for HDR.

\subsection{Potential for unintended off-target edits}

Programmable nucleases have high cleavage activity and show some degree of tolerance to mismatches for targetspecific binding. This creates the potential for unintended off-target events with the introduction of unwanted mutations somewhere in the genome ${ }^{[13]}$. In particular, Cas9-based editors have seen rapid improvements to increase binding specificity and associated reduction in off-target activity ${ }^{[14]}$. As a recent example, prime editors appear to be associated with much lower off-target editing compared to Cas9 nuclease ${ }^{[12]}$.

Systematic identification of all possible cleavage sites in vitro and their validation in an in vivo mouse model demonstrated that despite efficient editing, no detectable off-target mutations were found when following appropriate design guidelines for the gRNA/Cas9 editor ${ }^{[15]}$. PCR and sequencing analyses commonly characterize only short genomic regions, which might miss larger deletions and complex rearrangements that can also be introduced through DSB repair triggered by genome editing. This is an issue that is particularly relevant for multiplex applications ${ }^{[16]}$. Comprehensive unbiased genome-wide assessment of genome-edited sheep and goats showed that the level of de novo mutations was comparable to normal animals, with only one and two mutations attributable to off-target events, respectively ${ }^{[17,18]}$. Most studies to date observed no to negligible off-target effects in genomeedited livestock. Although the risk appears to be very small, the potential for off-target mutations should be considered.

\subsection{Editing somatic vs. embryonic cells}

Genome editing of cattle can be approached by two alternative experimental strategies. First, genome editors can be introduced into cultured primary somatic cells to effect site-specific sequence changes of their genomes. Following the isolation and characterization of cell clones, individual cells from cell clones that possess the intended edits can then be used as donor cells for somatic cell transfer (SCT) (Fig. 1(a)). This approach has so far been the method of choice for the generation of genome-edited cattle. It allows accurate characterization of the edited genotype, including monoallelic or biallelic modification and the potential introduction of any unintentional mutations from off-target events. Hence, it provides the ability to only generate cattle with intended genotypes and avoids the wasteful production of unwanted cattle. However, this approach is also associated with some major disadvantages. SCT is notoriously encumbered by low production efficiencies of viable offspring due to faulty or incomplete epigenetic reprogramming of the donor cell genome. This causes the commonly observed high rates of losses during pregnancy and can also affect the viability of live-born calves ${ }^{[19]}$. From a breeding perspective, adult somatic cloning increases the genetic lag between nucleus and commercial populations by one generation interval (equivalent to two years in cattle), compared to embryonic clones.

The second approach introduces genome editing tools into embryos, which are subsequently transferred into recipient cows for development to term and production of live genome-edited calves (Fig. 1(b)). As with somatic cells, not all embryos will be edited. To screen and identify edited embryos, there is the option to take a small biopsy which can provide a good representation about the editing status of the embryo and resulting animal ${ }^{[20]}$. The injection and biopsy do not compromise the developmental potential of the embryos.

Importantly, the same biopsy sample can be used for genomic selection, using SNP genotyping on different density bovine SNP BeadChips ${ }^{[21]}$. Following imputation with parental genotypes, there was high concordance $(\geqslant 95 \%)$ between biopsied and vitrified in vitro produced (IVP) embryos and their respective calf genotypes ${ }^{[21]}$. Applying this approach to dairy cattle, where vitrified IVP embryos are commonly used in breeding programs without loss of viability, would offer two advantages. First, it enables more intense selection of favorable genotypes within full-sib IVP embryos and second, it reduces the costs for yearling bulls and heifers because embryos of low genetic merit would not be transferred into surrogate mothers and reared to breeding age $\mathrm{e}^{[22]}$. Genome editing can effectively integrate with this embryo-based breeding platform, strongly decreasing the time to fixation for a desired allele compared to both genomic selection alone ${ }^{[23]}$ 
(a) Soma-mediated genome editing

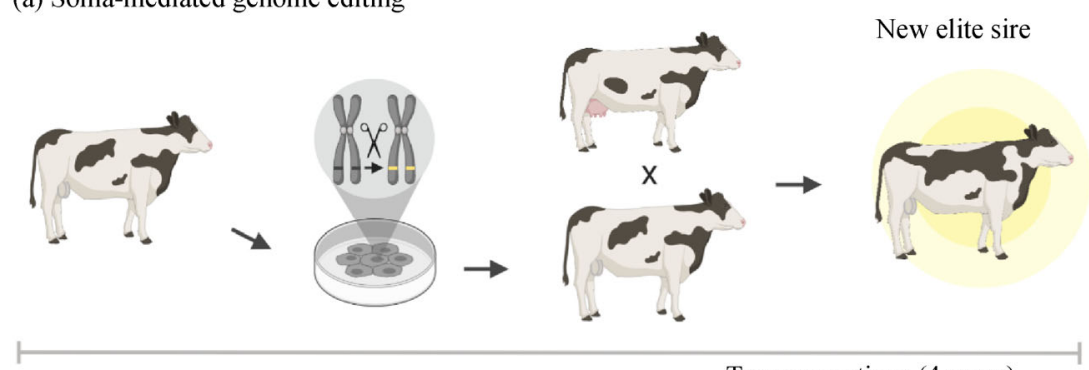

Two generations (4 years)

(b) Embryo-mediated genome editing

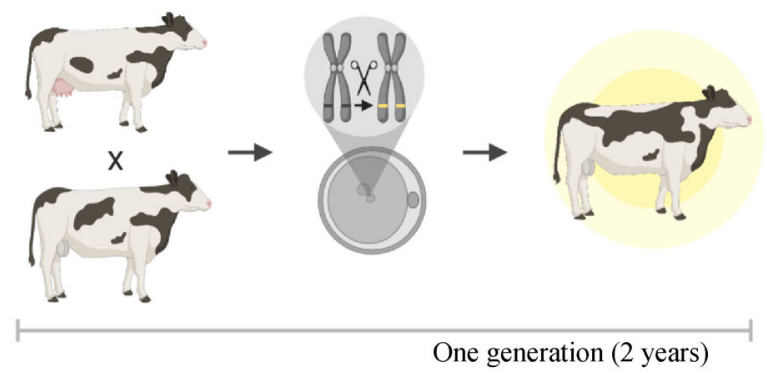

Fig. 1 Soma- and embryo-mediated genome editing approaches in cattle. (a) Somatic cells isolated from an elite sire are genome edited (locus black to gold) and cloned to introduce the modification on the same genetic background. A new elite sire with a higher breeding worth - and carrying the genomic modification - is produced after crossing the cloned sire with an elite dam. (b) For embryo-mediated genome editing, the elite sire and dam are crossed, and the genome editing carried out in the zygote, introducing the genomic modification in the higher breeding worth genetic background in a single generation.

and to somatic clones, which require another generation interval ${ }^{[24]}$. In contrast to the inefficient SCT route, embryo-mediated genome editing can therefore rapidly produce live genome-edited calves comparable to industry standards for conventional cattle. Thus, it fulfills an important prerequisite for a breeding application.

A major disadvantage of the embryo-mediated approach is the lack of control over the exact time and duration of nuclease and repair activities which can result in animals with variable degrees of mosaicism and one or several different mutated alleles (Table 1). Even with high HDR editing efficiencies, one could not completely rule out a low degree of mosaicism. Considering the large numbers of offspring that are produced from elite sires, any degree of mosaicism would pose a significant issue for breeding applications, as not all offspring would be of the desired genotype. However, this is not an unsurmountable problem and strategies to overcome mosaicism by embryonic cell transfer (ECT) are discussed in detail below.

\section{Embryo-mediated editing}

Genome edits have been introduced into eutherian zygotes, two-cell embryos or embryonic pluripotent stem cells (ePSCs), derived from high-value blastocysts. These embryo-mediated editing methods can be used indepen- dently or in combination. Zygotic and two-cell editing are two approaches that were already successfully applied in mouse and cattle, while bovine ePSC-mediated editing is still under development.

\subsection{Editing zygotes}

Editing at the earliest point in embryogenesis means that, in theory, all cells of the developing embryo would acquire the precise modification. There are few reports of zygotemediated genome editing in bovine embryos, differing in the type of nuclease used, as well as the timing, reagent and method of delivery (Table 1). A shared outcome from these diverse methods is the frequent production of mosaic embryos arising from several independent editing events from the one-cell stage onwards. These typically produce complex genetic mosaics, each with a unique spectrum of alleles and/or variable contribution of each allele to the whole animal, resulting in unpredictable and difficult-todefine phenotypes that necessitate further breeding of the founders ${ }^{[34]}$.

Earlier publications carrying out zygote-mediated genome editing relied on ZFN and TALENs instead of Cas9, probably a reflection of the long generation time of cattle vs. the rapid development of new editing tools. In fact, the only embryo-mediated HDR-edited cattle offspring so far have come from TALENs producing lactoglobulin-ablated 
Table 1 Publications of embryo-mediated genome editing in bovine zygotes

\begin{tabular}{|c|c|c|c|c|c|c|c|c|c|}
\hline Nuclease $^{a}$ & Reagent $^{\mathrm{b}}$ & $\begin{array}{l}\text { Delivery } \\
\text { method }^{\mathrm{c}}\end{array}$ & $\begin{array}{l}\text { Delivery time } \\
\text { (post IVF)/h }\end{array}$ & Target locus & $\begin{array}{l}\text { Intended } \\
\text { editing pathway }\end{array}$ & Edited embryos $/ \%$ & $\begin{array}{c}\text { Mosaic } \\
\text { embryos } / \%{ }^{\mathrm{f}}\end{array}$ & $\begin{array}{l}\text { Mosaic/total } \\
\text { edited offspring }\end{array}$ & Reference \\
\hline TALE & mRNA & CI & 19 & $\begin{array}{c}A C A N \text { or } \\
G D F 8\end{array}$ & NHEJ & NHEJ: $2-50$ & $\sim 20$ & - & {$[25]$} \\
\hline TALE & mRNA & $\mathrm{CI}$ & 24 & $G D F 8$ & NHEJ & NHEJ: $31-57$ & ND & $1 / 3$ & {$[26]$} \\
\hline $\begin{array}{l}\text { ZF or } \\
\text { TALE }\end{array}$ & $\begin{array}{l}\text { Plasmid or } \\
\text { mRNA }\end{array}$ & CI & 8 or 18 & $L G B$ & NHEJ \& HDR & $\begin{array}{l}\text { NHEJ: } 29-83 \\
\text { HDR: } 11-46\end{array}$ & $\sim 80$ & - & {$[27]$} \\
\hline TALE & plasmid & $\mathrm{CI}$ & 18 & $L G B$ & HDR & HDR: 21-32 & ND & $1 / 3^{g}$ & {$[20]$} \\
\hline Cas9 & mRNA & $\mathrm{CI}$ & $20-22$ & - & NHEJ & NHEJ: 83 & ND & - & {$[28]$} \\
\hline Cas9 & $\begin{array}{l}\text { Plasmid or } \\
\text { mRNA }\end{array}$ & CI & 5 & $P R N P$ & NHEJ \& HDR KI & $\begin{array}{c}\text { NHEJ: } 25-45 \\
\text { KI: } 12.5\end{array}$ & $\sim 85$ & - & [29] \\
\hline Cas9 & RNP & $\mathrm{E}$ & 8 & $P M E L$ & NHEJ \& HDR & $\begin{array}{c}\text { NHEJ: } 57-100 \\
\text { HDR: } 0-6\end{array}$ & ND & - & {$[30]$} \\
\hline Cas9 & RNP & $\mathrm{CI}$ & $\begin{array}{c}10 \text { (IVF), } \\
1 \text { (PG) }\end{array}$ & POU5F1 & NHEJ & NHEJ: 86 & $\sim 34$ & - & {$[31]$} \\
\hline Cas9 & RNP & $\mathrm{E}$ & $18-22$ & NANOS2 & NHEJ & NHEJ: $\sim 63$ & ND & - & {$[32]$} \\
\hline Cas9 & RNP & $\mathrm{E}$ & 10 or 15 & $G D F 8$ & NHEJ & NHEJ: $20-60$ & $\sim 75$ & - & [33] \\
\hline
\end{tabular}

Note: ${ }^{\mathrm{a}}$ Transcription activator-like effector (TALE), zinc finger (ZF). ${ }^{\mathrm{b}}$ Nuclease delivered as mRNA, plasmid, or preformed ribonucleoprotein (RNP) complex.

${ }^{c}$ Cytoplasmic injection (CI) or electroporation (E). ${ }^{\mathrm{d}}$ In vitro fertilized (IVF) or parthenogenetic (PG) embryos. ${ }^{\mathrm{e}}$ Non-homologous end joining (NHEJ), homologydirected repair (HDR), knockin (KI). ${ }^{\mathrm{f}}$ Normalized on the total number of edited embryos or not determined (ND). ${ }^{\mathrm{g}}$ Embryos were biopsied and screened for the presence of the HDR edit before transfer.

cattle ${ }^{[20]}$. Across all three DSB-based editing platforms, nucleases have been delivered as plasmid DNA, mRNA or as RNA/protein complexes (Table 1). Delivering the editing tools as plasmid DNA is convenient but carries the risk of unwanted insertions of plasmid vectors as has been observed in cattle that were edited for the Celtic polled mutation $^{[35]}$. Unwanted insertion can be avoided by delivering the editors in a different form and several studies have successfully demonstrated on-target mutagenesis in zygotes with preformed gRNA/Cas9 ribonucleoprotein (RNP) complexes ${ }^{[30-33]}$. Since designing gRNAs for Cas9 is far simpler than TALENs or ZFNs, delivering preformed gRNA/Cas9 RNP complexes is likely to be the preferred option for future accelerated breeding programs.

The standard method of delivery is via cytoplasmic, rather than pronuclear, microinjection (Fig. 2). This

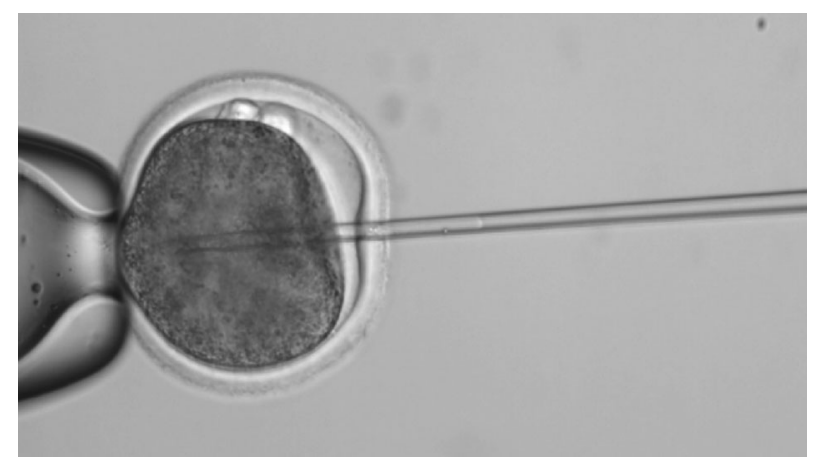

Fig. 2 Cytoplasmic injection in a bovine zygote. Image courtesy of Dr. Jingwei Wei (AgResearch, Ruakura Research Centre, New Zealand). preference is due to the high lipid content in bovine oocytes and zygotes, which obscures visibility of the pronuclei by standard transmitted-light microscopy. Cytoplasmic injection is near $100 \%$ efficient and the associated plasma membrane damage and volume change is transient and generally well-tolerated by the zygote. Provided that the dimensions of the injection capillaries and injection parameters (e.g., injection pressure and duration) are standardized, this technique results in reproducible amounts of material being delivered. However, this method relies on high technical skills, introducing operator-dependent experimental variation as a confounding parameter in editing experiments. It also requires expensive micromanipulation, microscopy and pipette fabrication equipment and is relatively low-throughput.

By contrast, electroporation offers a higher throughput alternative, which is less labor- and cost-intensive. It can be simultaneously applied to many zygotes and only needs a stereomicroscope, electroporator and fusion chamber. This approach has been reported to deliver gRNA/Cas9 RNPs, Cas9 mRNA and gRNAs into rodent embryos, achieving high biallelic editing efficiencies ${ }^{[36,37]}$. This was followed by the validation of electroporation as a suitable method to edit bovine embryos ${ }^{[32,33]}$. In addition, the feasibility was demonstrated to HDR-edit bovine zygotes by electroporating gRNA/Cas9 RNPs and an exogenous repair template ${ }^{[30]}$.

Multiple time points during the first bovine cell cycle have been evaluated for NHEJ or HDR editing efficiency. Most reports in cattle have focused on introducing NHEJ mutations for simple loss-of-function experiments, with nuclease delivery time ranging from 5 to $24 \mathrm{~h}$ after in vitro 
fertilization (IVF) (Table 1). At each of these time points, moderate editing efficiencies have been achieved with usually at least half of the embryos carrying NHEJ generated indel mutations. However, fewer publications have assessed HDR-based editing in bovine embryos.

HDR occurs at higher frequency during late $S$ and $G_{2}$ phase of the cell cycle ${ }^{[38]}$. So, timing the delivery of the editing tools may be important for achieving precise HDR editing. In bovine zygotes, $G_{1}$ phase lasts from about 6-14 $\mathrm{h}$ post-IVF, S-phase starts at $11-15 \mathrm{~h}$ and ends at 23$26 \mathrm{~h}$, followed by a short $\mathrm{G}_{2}$-phase at $26-31 \mathrm{~h}$ and the first cleavage divisions after $26 \mathrm{~h}^{[39]}$. For HDR editing in bovine embryos, the nuclease and repair template were either co-delivered before (5-10 h post-IVF) or during $\mathrm{mid} / \mathrm{late} \mathrm{S}$ phase (18-24 h post-IVF, Table 1). When delivered in $\mathrm{G}_{1}$, cleavage and repair of the target sites will result in edits that are replicated in both DNA strands during subsequent $\mathrm{S}$ phase. By contrast, editing during $\mathrm{S}$ phase occurs while each chromosomal DNA strand is being replicated into two sister chromatids, doubling the number of target sites, but coinciding with the DSB repair machinery being most active ${ }^{[38]}$. Previous work by our group showed that there was no significant difference in the proportion of HDR-edited embryos between delivering the nuclease and repair template at $\mathrm{G}_{1}(8 \mathrm{~h})$ vs. S phase $(18 \mathrm{~h})^{[27]}$. These experiments were mostly performed with ZFNs delivered as a plasmid, with the DNA cleavage expected to be faster if the nuclease was delivered as a protein. Further experiments are required to optimize delivery time for HDR when using the more convenient gRNA/Cas9 RNPs. Since mosaic embryos with more than two alleles are commonly observed, the editing could be continuing throughout subsequent cleavage divisions ${ }^{[40]}$.

HDR editing efficiency in zygotes is relatively low and was reported to be in the range of $11 \%-46 \%{ }^{[27]}$ and $21 \%$ $32 \%^{[20]}$ of cytoplasmically injected zygotes for the introduction of a 9-bp deletion in the bovine $L G B$ gene. By electroporation, the efficiencies were even lower with only up to $6 \%$ of embryos showing some degree of HDR editing ${ }^{[30]}$. For large transgene knockin experiments, Bevacqua et al. ${ }^{[29]}$ reported that Cas9 improved targeted insertion with one of eight embryos correctly modified compared to zero of 175 when using just the repair template. This shows that direct HDR editing is possible in bovine zygotes, but the reported efficiencies would need to be greatly increased or correctly edited embryos identified and selectively multiplied in order to be useful for a breeding program.

\subsection{Optimizing HDR outcomes}

HDR efficiencies still remain low, especially for introducing large DNA sequences. A number of approaches have been devised to shift the balance between competing NHEJ and HDR pathways toward the latter. These include the use of chemical and biochemical compounds to interfere with the DNA repair machinery - either by blocking the NHEJ pathway, with DNA-dependent protein kinase inhibitors like M3814 $4^{[41]}$, or by stimulating the HDR pathway, with mRNA from HR effectors like RAD51 ${ }^{[42]}$ or with stimulating molecules like RS-1 ${ }^{[43]}$. As pharmacological inhibitors can be cytotoxic and yield variable efficiency, HDR can also be enhanced by coupling Cas9 expression to cell cycle effectors at the late $\mathrm{S}$ to $\mathrm{G} 2$ phase $^{[44,45]}$ or by tethering the DNA template to the gRNA/Cas9 RNP complex, juxtaposing the template DNA to the DSB repair site $^{[46-48]}$. In addition, HDR repair efficiency depends on how the DNA template is delivered: as a single-stranded DNA molecule ${ }^{[49]}$, as recombinant adeno-associated viral vector $^{[50]}$, or in a cassette designed to trigger alternative DNA repair pathways, like microhomology-mediated end joining ${ }^{[51]}$, homology-mediated end joining ${ }^{[52]}$, or homology-independent targeted integration ${ }^{[53]}$.

Direct delivery into embryos provides an additional opportunity to improve HDR outcomes. In contrast to zygotic injection, much higher HDR-mediated knockin efficiencies were achieved by cytoplasmic microinjection into two-cell mouse embryos, combined with a modified streptavidin-biotin Cas9 system ${ }^{[46]}$. This strategy resulted in a 10-fold increased knockin efficiency compared to standard methods, higher frequency of homozygous insertions, and increased germline contribution. Benefits were attributed to two factors. First, the two-cell stage marks the onset of major embryonic genome activation in the mouse, which is associated with increased open chromatin that may improve the accessibility for Cas9 to the target site. Second, mouse embryos display an exceptionally long $\mathrm{G}_{2}$ cell cycle phase during the twocell stage, extending the time for HDR-mediated repair to occur. The same approach is less likely to be successful in cultured bovine embryos, where embryonic genome activation occurs at the eight-cell stage ${ }^{[54]}$, concomitant with the longest cell cycle duration at this stage ${ }^{[55]}$. Compared to zygote delivery, introducing editors into all blastomeres at the two- or eight-cell stage likely increases mosaicism, as there are now multiple independent genome editing events that need to resolve in the developing embryo. Also, transfecting multiple blastomeres, either by injection or electroporation, is technically more difficult, which makes its implementation less straightforward.

All the above optimization strategies have so far been unable to sufficiently lift HDR efficiencies to make precision genome editing in embryos for breeding a realistic proposition. Without future improvements, multiplication of HDR-edited but rare embryos might offer another solution.

\section{Multiplying edited embryonic genotypes}

The probability of obtaining a viable calf from a single edited IVF embryo is about $45 \%{ }^{[56]}$. To address this 
biological limit, different strategies for multiplying edited genomic selection embryos are being developed. These also address low editing efficiency by identifying and selectively multiplying correctly targeted embryonic cells. For zygote-mediated editing, serial cloning from early cleavage-stage or morula blastomeres can multiply the desired genotype, resolve potential mosaicism and ensure the generation of live animals. For ePSC editing, robust in vitro proliferation enables genotype amplification and characterization before potentially generating a large number of animals.

\subsection{Blastomeres}

The zygote cleaves into progressively smaller nucleated cells, termed blastomeres, without increasing total embryo volume ${ }^{[57]}$. This process ends at the blastocyst stage when cells initiate growth and replicative proliferation for the first time during embryo development ${ }^{[57]}$. Until the eightcell stage, bovine blastomeres are still totipotent, i.e., able to give rise to all cell types on their own, and genotype multiplication can be achieved by embryo splitting ${ }^{[58]}$. Following zygote-mediated editing, dissociating totipotent blastomeres at the two- to eight-cell stage could potentially resolve early embryonic mosaicism by segregating the different genotypes generated at the one-cell stage ${ }^{[59]}$. Multiplication of the edited genotype is maximally two to 8 -fold with this approach, not accounting for some blastomeres that must be sacrificed for genomic selection and validation of edits. This limits the applicability of blastomere splitting for resolving mosaicism and genotype amplification.

The pre-compacting morula stage (32-64 cells) offers an intermediate starting point for embryo multiplication between early cleavage-stage stages, which are limited in numbers, and blastocyst-derived ePSCs, which can produce large numbers but have not been routinely established in cattle. Biopsy, genotyping and vitrification procedures, originally developed for genomic selection of blastocysts ${ }^{[22]}$, can be applied to identify the best morula genotypes $^{[60]}$. Modest genotype multiplication by ECT is feasible with morula blastomeres and editing is already fixed because introduced editors are unlikely to still be active at this stage ${ }^{[40]}$. As each embryo derives from a single donor cell, ECT segregates edited from unedited donors and only generates non-mosaic embryos (Fig. 3). Hence, genomically selected morulae can be dissociated into single donor cells for ECT, resulting in genotype amplification and segregation. In principle, several rounds of iterative ECT can achieve further multiplication by serial embryo recycling and intermittent cryopreservation. Importantly, ECT multiplies both edited and unedited elite embryos.

\subsection{Blastocysts and ePSCs}

Blastocysts can be microsurgically bisected, but the biological multiplication factor to term is low $(\sim 1.2$ times) due to the compromised in vivo survival of demiembryos $^{[61]}$. Instead, blastocyst-derived ePSCs offer a promising alternative for both multi-editing (see below) and multiplication. Under the right culture conditions, these cells remain capable, at the single cell level, to produce all adult cell types, including functional gametes $^{[62,63]}$. This ability is retained even after extensive genetic modification by homologous recombination ${ }^{[64]}$ or genome editing ${ }^{[65]}$. Only cells capable of germline transmission or tetraploid complementation, wherein ePSCs are introduced into a tetraploid host embryo and forced to form the entire animal ${ }^{[66]}$, are considered fully pluripotent. Such cells have been referred to as naïve $\mathrm{PSCs}^{[67]}$. Naïve PSCs are capable of extended self-renewal and biological amplification of a selected genotype without genetic transformation. Their high clonogenicity from single cells allows maintaining stable diploid cultures over hundreds of population doublings ${ }^{[68]}$. This would be highly desirable for in vitro multiplication of rare highvalue embryonic genotypes.

Ever since ePSCs were first described in mice, there has been an interest to derive analogous cells from farm animals, however, all attempts to isolate naïve bovine ePSCs have so far been unsuccessful ${ }^{[69]}$. Progress in

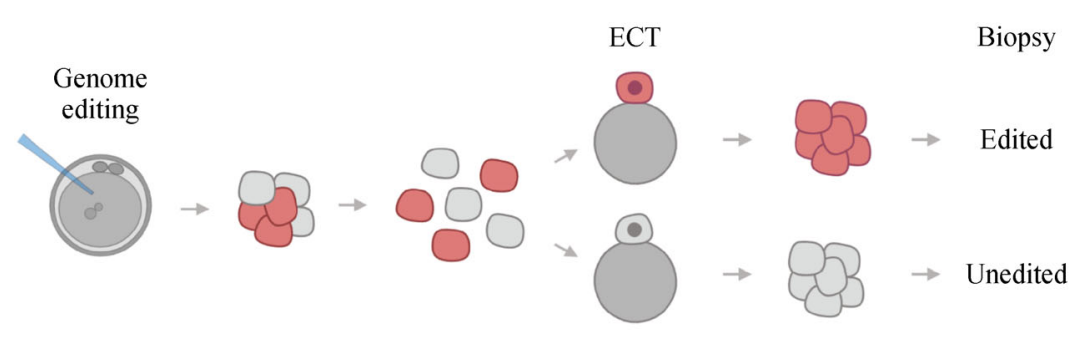

Fig. 3 Resolving mosaicism in edited embryos by embryonic cell transfer (ECT) blastomere cloning. After genome editing in the zygote, a mosaic morula can be dissociated and the blastomeres used for ECT. The resulting embryos derived from a single blastomere would then be non-mosaic, which can be confirmed with an embryo biopsy before transfer or cryopreservation. 
understanding of pluripotency regulation in mice and humans, combined with extensive chemical screening, has led to the development of chemically defined culture conditions that yielded expanded potential stem cells in pigs $^{[70]}$. These cells show molecular and functional features of naïve pluripotency, including expression of key pluripotency genes and permissiveness to genome editing. Initial plating and cell line derivation efficiency from individual blastocysts was only averaging $\sim 30 \%$ but, once established, porcine ePSCs reached $>40$ passages without overt differentiation, while remaining karyotypically normal ${ }^{[70]}$. Similar results were also reported for bovine ePSC-like cells, achieving $>50$ passages with stable population-doubling times and normal karyotypes $^{[71]}$. Importantly, porcine ePSCs also engendered all embryonic and extra-embryonic somatic cell lineages, but so far not the germline, in chimaeras. These advances suggest that the naïve pluripotent state may be a conserved feature of mammalian embryogenesis that could be effectively captured, without genetic intervention, in other livestock species.

\subsection{Multiplex editing of ePSCs}

The majority of important livestock traits are complex, quantitative and controlled by multiple genes. Their manipulation will require multiplex genomic modifications, which are likely to be challenging to achieve by zygotic injection alone. Long-term proliferating livestock ePSCs, once available, would present a practical alternative to zygote-mediated editing (Fig. 3).

In mouse and human ePSCs, the pluripotent cell state facilitates transgenesis, homologous recombination and genome editing with ZFNs and TALENs ${ }^{[72-74]}$. Likewise, Cas9 allows for fast and efficient multiplex mutagenesis at endogenous genomic loci in human ${ }^{[75]}$ and mouse $\mathrm{PSCs}^{[65]}$. Even though biallelic HDR frequencies in human PSCs are generally 5-20-fold lower than in tumor cell lines, this can be overcome by positive selection strategies, efficient subcloning of cells with rare editing events or enhanced delivery methods for nuclease and template $^{[75]}$. Similarly, transfected mouse PSCs can be enriched by either using drug selection or sorting for the expression of fluorescent reporters. Since ePSCs are highly clonogenic under defined long-term culture conditions, single cells can be expanded into sufficient numbers of primary colonies to allow for thorough characterization of the introduced edits (Fig. 4). At the same time, ePSC clonal strains would resolve any potential mosaicism present in the founder population. Clonal strains derived from unedited cells that have undergone the same targeting process provide isogenic parental control cells.

While simultaneous editing is possible, its scope will be limited by the number of simultaneous DSBs in the genome that a cell can tolerate for survival. By contrast, the indefinite growth potential of ePSCs allows for essentially unlimited sequential editing and engineering of compound genotypes optimally suited to study complex biological processes. In livestock, this would facilitate stacking of beneficial causative variants into an elite genetic background in a single generation, short-cutting decades of traditional crossbreeding and backcrossing to generate enhanced phenotypes.

\section{Generating genome-edited animals from embryonic genotypes}

\subsection{Clones}

The two main reproductive strategies for producing animals from either edited embryos or naïve ePSCs are (1) ECT cloning, and (2) germline chimaera formation (Fig. 5). In ECT cloning, the incidence and severity of abnormal phenotypes is greatly reduced with blastomere compared to somatic clones ${ }^{[76,77]}$. It remains to be shown that this also holds true for edited blastomeres, multiplied by serially cloning, but potentially this approach can substantially lessen animal welfare burdens. The situation is less clear with cloning from ePSCs. In mouse cloning experiments, ePSCs did not perform significantly better than genetically matched somatic cells ${ }^{[78]}$. However, mouse ePSC often contain non-reprogrammable karyotypic abnormalities ${ }^{[79]}$ and are epigenetically unstable in long-term culture during targeting experiments ${ }^{[80]}$, complicating assessment of their true reprogramming potential. This has prompted the notion that short-term cultures of primary ePSCs, which are more likely to be devoid of

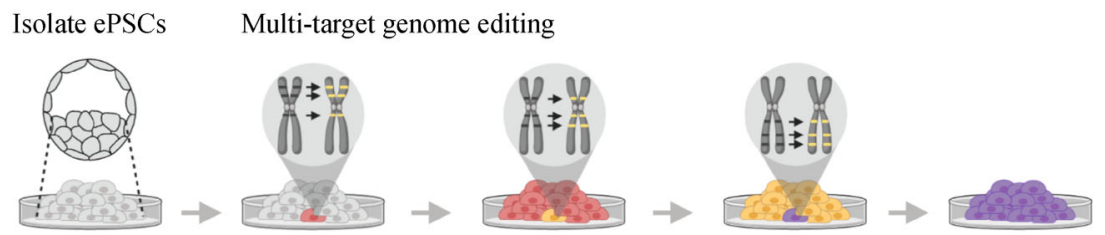

Fig. 4 Multiplex genome editing in embryonic pluripotent stem cells (ePSCs) isolated from a genomically selected elite blastocyst. With high clonogenicity and stable pluripotency, ePSCs would allow sequential multiplex genome editing of multiple loci (loci black to gold), followed by clonal expansion of edited strains. 


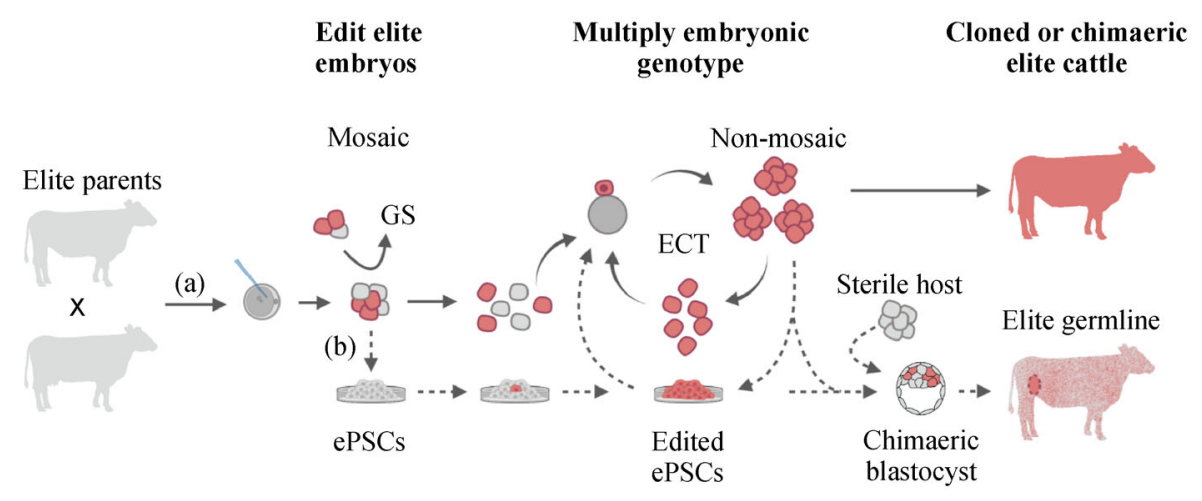

Fig. 5 Embryo-mediated accelerated breeding in cattle. (a) Using proven technologies (solid arrows), precise genomic edits can be introduced in zygotes from elite sires and the best embryos identified by genomic selection (GS). The desired embryonic genotype can then be multiplied by embryonic cell transfer (ECT) cloning and elite offspring generated. (b) A complementary pathway (dashed arrows) is centered around embryonic pluripotent stem cells (ePSCs). ePSCs would be isolated from an elite embryo and numerous genomic modifications introduced. Alternatively, ePSCs would be isolated from embryos multiplied by ECT for subsequent genome editing. Offspring could then be produced by cloning or as a chimaera with absolute transmission of the elite germline.

genetic and epigenetic aberrations, may result in higher cloning efficiency, similar to the results with embryonic blastomeres $^{[78]}$.

\subsection{Germline chimaeras}

Chimaera formation, on the other hand, involves introducing donor blastomeres or naïve ePSCs into a normal diploid host embryo of a different zygotic origin, resulting in widespread somatic and germline contribution of the donor cells. Since the donor germline contribution is seldom $100 \%$, only some of the offspring originate from the donor genotype, necessitating time-consuming crossbreeding of the founder generation, which renders this approach impractical for livestock breeding. To overcome this problem, it is possible to genetically ablate the host germline ${ }^{[81-85]}$, providing a suitable vehicle for exclusive transmission of the donor haplotype, either from transplanted spermatogonial stem cells ${ }^{[86]}$ or embryonic cells developing into gametes. The latter approach relies on embryo complementation of germline-deficient hosts, a mechanism that has, in principle, been demonstrated as feasible in sheep ${ }^{[87]}$, cattle ${ }^{[88,89]}$ and pigs ${ }^{[90,91]}$. The generation of such absolute transmitters with exclusively embryo-derived elite donor germlines provides an exciting opportunity for accelerating genetic gain ${ }^{[22]}$. Absolute transmitters could functionally replace somatic clones, potentially reducing cloning-associated animal welfare problems.

\subsection{In vitro gametogenesis}

It may also be possible to differentiate edited diploid ePSCs into haploid male and female gametes, similar to what has been achieved in mice ${ }^{[92]}$. Depending on the time lines for cell differentiation, meiotic recombination and proliferation, this approach, originally termed whizzogenetics, could theoretically shorten the generation interval, provided that the same selection accuracy can be maintained ${ }^{[93]}$. Even though the biological and practical hurdles for reconstituting gametogenesis in the dish are enormous, this new technology may provide an alternative source of gametes for in vitro breeding in the future.

\section{Regulatory aspects}

Genome editing is a relatively new technology that is associated with regulatory uncertainty. Regulations established for the control of transgenic technology appear no longer appropriate for the level of precision and control that is possible with the new editing technology and is a topic of intense international discussion ${ }^{[94]}$. While many countries are still developing their final position, economic heavyweights like the EU and the USA have opted to regulate genome-edited animals just like transgenic animals even though genome-edited animals can be indistinguishable from naturally-occurring animals ${ }^{[95,96]}$. However, there are also a few countries that have taken a different path. The regulations in Argentina, Australia and Brazil reflect the reduced risk profiles of genome-edited animals and regulation is not required if genome-edited animals do not contain any foreign $\mathrm{DNA}^{[97,98]}$. The simplest regulatory approach for new technologies has probably been implemented by Canada ${ }^{[99]}$. The trigger for regulation is not a new technology but rather a new animal trait, irrespective of what technology was used to create that trait. Realizing the great potential of genome-edited animals toward sustainable solutions for pressing environmental and food security issues will only be possible if more countries adopt risk-adjusted regulations that might allow the widespread use of such animals. 


\section{Conclusions}

Almost two decades ago it was predicted that embryobased genome selection, modification and multiplication would replace somatic cell-based approaches to accelerate livestock breeding ${ }^{[100]}$. With the advent of accurate marker- and sequence-based embryo selection, precise multi-editing and more sophisticated serial embryo multiplication, this prediction is now getting closer to reality (Fig. 5). Conceptually, some key advantages of embryomediated approaches for genetic improvement have not changed since then: (1) genomically-selected embryos capture the most recent genetic gains, compared to the genetic lag associated with adults; (2) embryonic cells are amenable to complex genomic modifications owing to their extensive proliferation potential; (3) embryonic donors are inherently easier to reprogram, resulting in greater cloning efficiency, reduced animal production costs and animal welfare issues; and (4) animal clones may even be replaced with chimaeras if complementation with embryonic donors can efficiently establish absolute germline transmitters. In addition, genome editing can selectively introduce naturally-occurring beneficial variants into various elite genetic backgrounds in a single generation without leaving foreign DNA in the targeted genome.

To use genome editing productively for livestock improvement, putative causative variants for desirable traits must first be identified, emphasizing the ongoing need for large-scale gene discovery programs. Genome editing can then determine causality of the most promising selection candidates before their embryo-meditated introgression for animal production. Stacking multiple edited variants in the most recent elite genetic background would rapidly generate fitter, healthier and more sustainable animals, required to reduce our vulnerabilities to diminishing natural resources and secure sustainable livestock production.

Acknowledgements This work was funded by AgResearch and the Ministry of Business, Innovation and Employment. Figures containing graphic art were created with Biorender.com.

Compliance with ethics guidelines Zachariah McLean, Björn Oback, and Götz Laible declare that they have no conflicts of interest or financial conflicts to disclose.

This article is a review and does not contain any studies with human or animal subjects performed by any of the authors.

\section{References}

1. Thornton $P$ K. Livestock production: recent trends, future prospects. Philosophical Transactions of the Royal Society of London. Series B: Biological Sciences, 2010, 365(1554): 28532867

2. Meuwissen T, Hayes B, Goddard M. Genomic selection: a paradigm shift in animal breeding. Animal Frontiers, 2016, 6(1): $6-14$
3. Meuwissen T H E, Hayes B J, Goddard M E. Prediction of total genetic value using genome-wide dense marker maps. Genetics, 2001, 157(4): 1819-1829

4. Tait-Burkard C, Doeschl-Wilson A, McGrew M J, Archibald A L, Sang H M, Houston R D, Whitelaw C B, Watson M. Livestock 2.0genome editing for fitter, healthier, and more productive farmed animals. Genome Biology, 2018, 19(1): 204

5. Georges M. Towards sequence-based genomic selection of cattle. Nature Genetics, 2014, 46(8): 807-809

6. Hauschild-Quintern J, Petersen B, Cost G J, Niemann H. Gene knockout and knockin by zinc-finger nucleases: current status and perspectives. Cellular and Molecular Life Sciences, 2013, 70(16): 2969-2983

7. Mussolino C, Cathomen T. TALE nucleases: tailored genome engineering made easy. Current Opinion in Biotechnology, 2012, 23(5): 644-650

8. Sander J D, Joung J K. CRISPR-Cas systems for editing, regulating and targeting genomes. Nature Biotechnology, 2014, 32(4): 347-355

9. Xiao A, Wang Z X, Hu Y Y, Wu Y D, Luo Z, Yang Z P, Zu Y, Li W Y, Huang P, Tong X J, Zhu Z Y, Lin S, Zhang B. Chromosomal deletions and inversions mediated by TALENs and CRISPR/Cas in zebrafish. Nucleic Acids Research, 2013, 41(14): e141

10. Tan W F, Proudfoot C, Lillico S G, Whitelaw C B A. Gene targeting, genome editing: from Dolly to editors. Transgenic Research, 2016, 25(3): 273-287

11. Eid A, Alshareef S, Mahfouz M M. CRISPR base editors: genome editing without double-stranded breaks. Biochemical Journal, 2018, 475(11): 1955-1964

12. Anzalone A V, Randolph P B, Davis J R, Sousa A A, Koblan L W, Levy J M, Chen P J, Wilson C, Newby G A, Raguram A, Liu D R. Search-and-replace genome editing without double-strand breaks or donor DNA. Nature, 2019, 576(7785): 149-157

13. Carroll D. Staying on target with CRISPR-Cas. Nature Biotechnology, 2013, 31(9): 807-809

14. Komor A C, Badran A H, Liu D R. CRISPR-based technologies for the manipulation of eukaryotic genomes. Cell, 2017, 168(1-2): $20-36$

15. Akcakaya P, Bobbin M L, Guo J A, Malagon-Lopez J, Clement K, Garcia S P, Fellows M D, Porritt M J, Firth M A, Carreras A, Baccega T, Seeliger F, Bjursell M, Tsai S Q, Nguyen N T, Nitsch R, Mayr L M, Pinello L, Bohlooly-Y M, Aryee M J, Maresca M, Joung J K. In vivo CRISPR editing with no detectable genomewide off-target mutations. Nature, 2018, 561(7723): 416-419

16. Kosicki M, Tomberg K, Bradley A. Repair of double-strand breaks induced by CRISPR-Cas9 leads to large deletions and complex rearrangements. Nature Biotechnology, 2018, 36(8): 765-771

17. Li C, Zhou S W, Li Y, Li G W, Ding Y G, Li L, Liu J, Qu L, Sonstegard T, Huang X X, Jiang Y, Chen Y L, Petersen B, Wang X L. Trio-based deep sequencing reveals a low incidence of off-target mutations in the offspring of genetically edited goats. Frontiers in Genetics, 2018, 9: 449

18. Wang X L, Liu J, Niu Y Y, Li Y, Zhou S W, Li C, Ma B H, Kou Q F, Petersen B, Sonstegard T, Huang X X, Jiang Y, Chen Y L. Low incidence of SNVs and indels in trio genomes of Cas9-mediated multiplex edited sheep. BMC Genomics, 2018, 19(1): 397 
19. Wells D N. Animal cloning: problems and prospects. Revue Scientifique et Technique (International Office of Epizootics), 2005, 24(1): 251-264

20. Wei J W, Wagner S, Maclean P, Brophy B, Cole S, Smolenski G, Carlson D F, Fahrenkrug S C, Wells D N, Laible G. Cattle with a precise, zygote-mediated deletion safely eliminate the major milk allergen beta-lactoglobulin. Scientific Reports, 2018, 8(1): 7661

21. Mullaart E, Wells D. Embryo Biopsies for Genomic Selection. In: Niemann H, Wrenzycki C, eds. Animal Biotechnology 2: Emerging Breeding Technologies. Cham: Springer, 2018, 81-94

22. Hely F S, Amer P R, Oback B, Wells D N. Integration of IVF technologies with genomic selection to generate high merit AI bulls: a simulation study. Proceedings of the Association for the Advancement of Animal Breeding and Genetics, 2017, 22: 533536

23. Bastiaansen J W M, Bovenhuis H, Groenen M A M, Megens H J, Mulder H A. The impact of genome editing on the introduction of monogenic traits in livestock. Genetics, Selection, Evolution, 2018, 50(1): 18

24. Jenko J, Gorjanc G, Cleveland M A, Varshney R K, Whitelaw C B A, Woolliams J A, Hickey J M. Potential of promotion of alleles by genome editing to improve quantitative traits in livestock breeding programs. Genetics, Selection, Evolution, 2015, 47(1): 55

25. Carlson D F, Tan W, Lillico S G, Stverakova D, Proudfoot C, Christian M, Voytas D F, Long C R, Whitelaw C B A, Fahrenkrug $\mathrm{S}$ C. Efficient TALEN-mediated gene knockout in livestock. Proceedings of the National Academy of Sciences of the United States of America, 2012, 109(43): 17382-17387

26. Proudfoot C, Carlson D F, Huddart R, Long C R, Pryor J H, King T J, Lillico S G, Mileham A J, McLaren D G, Whitelaw C B A, Fahrenkrug S C. Genome edited sheep and cattle. Transgenic Research, 2015, 24(1): 147-153

27. Wei J W, Wagner S, Lu D, Maclean P, Carlson D F, Fahrenkrug S C, Laible G. Efficient introgression of allelic variants by embryomediated editing of the bovine genome. Scientific Reports, 2015, 5 (1): 11735

28. Bogliotti Y S, Vilarino M, Ross P J. Laser-assisted cytoplasmic microinjection in livestock zygotes. Journal of Visualized Experiments, 2016, 116: e54465

29. Bevacqua R J, Fernandez-Martin R, Savy V, Canel N G, Gismondi M I, Kues W A, Carlson D F, Fahrenkrug S C, Niemann H, Taboga O A, Ferraris S, Salamone D F. Efficient edition of the bovine PRNP prion gene in somatic cells and IVF embryos using the CRISPR/Cas9 system. Theriogenology, 2016, 86(8): 1886-1896. e1

30. Brophy B, Cole S, Gaynor P, Laible G, Oback B, Wei J. Developing the conditions for bovine zygote-mediated genome editing by electroporation. In: Proceedings of the World Congress on Genetics Applied to Livestock Production, Technologies: Gene Editing. World Congress on Genetics Applied to Livestock Production Digital Archive, 2018, 1118

31. Daigneault B W, Rajput S, Smith G W, Ross P J. Embryonic POU5F1 is required for expanded bovine blastocyst formation. Scientific Reports, 2018, 8(1): 7753

32. Miao D Q, Giassetti M I, Ciccarelli M, Lopez-Biladeau B, Oatley J M. Simplified pipelines for genetic engineering of mammalian embryos by CRISPR-Cas9 electroporation. Biology of Reproduction, 2019, 101(1): 177-187

33. Namula Z, Wittayarat M, Hirata M, Hirano T, Nguyen N T, Le Q A, Fahrudin M, Tanihara F, Otoi T. Genome mutation after the introduction of the gene editing by electroporation of Cas9 protein (GEEP) system into bovine putative zygotes. In vitro Cellular \& Developmental Biology - Animal, 2019, 55(8): 598-603

34. Teboul L, Murray S A, Nolan P M. Phenotyping first-generation genome editing mutants: a new standard? Mammalian Genome, 2017, 28(7-8): 377-382

35. Young A E, Mansour T A, McNabb B R, Owen J R, Trott J F, Brown C T, Van Eenennaam A L. Genomic and phenotypic analyses of six offspring of a genome-edited hornless bull. Nature Biotechnology, 2019 [Published Online] doi: 10.1038/s41587019-0266-0

36. Kaneko T, Sakuma T, Yamamoto T, Mashimo T. Simple knockout by electroporation of engineered endonucleases into intact rat embryos. Scientific Reports, 2015, 4(1): 6382

37. Hashimoto M, Yamashita Y, Takemoto T. Electroporation of Cas9 protein/sgRNA into early pronuclear zygotes generates nonmosaic mutants in the mouse. Developmental Biology, 2016, 418 (1): $1-9$

38. Hustedt N, Durocher D. The control of DNA repair by the cell cycle. Nature Cell Biology, 2017, 19(1): 1-9

39. Laurinčík J, Hyttel P, Baran V, Eckert J, Lucas-Hahn A, Pivko J, Niemann H, Brem G, Schellander K. A detailed analysis of pronucleus development in bovine zygotes in vitro: cell-cycle chronology and ultrastructure. Molecular Reproduction and Development, 1998, 50(2): 192-199

40. Ma H, Marti-Gutierrez N, Park S W, Wu J, Lee Y, Suzuki K, Koski A, Ji D, Hayama T, Ahmed R, Darby H, Van Dyken C, Li Y, Kang E, Park A R, Kim D, Kim S T, Gong J H, Gu Y, Xu X, Battaglia D, Krieg S A, Lee D M, Wu D H, Wolf D P, Heitner S B, Belmonte J C I, Amato P, Kim J S, Kaul S, Mitalipov S. Correction of a pathogenic gene mutation in human embryos. Nature, 2017, 548 (7668): 413-419

41. Riesenberg S, Chintalapati M, Macak D, Kanis P, Maricic T, Pääbo S. Simultaneous precise editing of multiple genes in human cells. Nucleic Acids Research, 2019, 47(19): e116

42. Wilde J J, Aida T, Wienisch M, Zhang Q G, Qi P M, Feng G P. Efficient zygotic genome editing via RAD51-enhanced interhomolog repair. bioRxiv, 2018 [Preprint] doi: 10.1101/263699

43. Song J, Yang D S, Xu J, Zhu T Q, Chen Y E, Zhang J F. RS-1 enhances CRISPR/Cas9- and TALEN-mediated knock-in efficiency. Nature Communications, 2016, 7(1): 10548

44. Gutschner T, Haemmerle M, Genovese G, Draetta G F, Chin L. Post-translational regulation of Cas9 during G1 enhances homology-directed repair. Cell Reports, 2016, 14(6): 1555-1566

45. Howden S E, McColl B, Glaser A, Vadolas J, Petrou S, Little M H, Elefanty A G, Stanley E G A. Cas9 variant for efficient generation of indel-free knockin or gene-corrected human pluripotent stem cells. Stem Cell Reports, 2016, 7(3): 508-517

46. Gu B, Posfai E, Rossant J. Efficient generation of targeted large insertions by microinjection into two-cell-stage mouse embryos. Nature Biotechnology, 2018, 36(7): 632-637

47. Ma M, Zhuang F F, Hu X B, Wang B L, Wen X Z, Ji J F, Xi J J. 
Efficient generation of mice carrying homozygous double-floxp alleles using the Cas9-Avidin/Biotin-donor DNA system. Cell Research, 2017, 27(4): 578-581

48. Aird E J, Lovendahl K N, St. Martin A S, Harris R S, Gordon W R. Increasing Cas9-mediated homology-directed repair efficiency through covalent tethering of DNA repair template. Communications Biology, 2018, 1(1): 54

49. Quadros R M, Miura H, Harms D W, Akatsuka H, Sato T, Aida T, Redder R, Richardson G P, Inagaki Y, Sakai D, Buckley S M, Seshacharyulu P, Batra S K, Behlke M A, Zeiner S A, Jacobi A M, Izu Y, Thoreson W B, Urness L D, Mansour S L, Ohtsuka M, Gurumurthy C B. Easi-CRISPR: a robust method for one-step generation of mice carrying conditional and insertion alleles using long ssDNA donors and CRISPR ribonucleoproteins. Genome Biology, 2017, 18(1): 92

50. Gaj T, Epstein B E, Schaffer D V. Genome engineering using adeno-associated virus: basic and clinical research applications. Molecular Therapy, 2016, 24(3): 458-464

51. Nakade S, Tsubota T, Sakane Y, Kume S, Sakamoto N, Obara M, Daimon T, Sezutsu H, Yamamoto T, Sakuma T, Suzuki K T. Microhomology-mediated end-joining-dependent integration of donor DNA in cells and animals using TALENs and CRISPR/ Cas9. Nature Communications, 2014, 5(1): 5560

52. Yao X, Wang X, Hu X D, Liu Z, Liu J L, Zhou H B, Shen X W, Wei Y, Huang Z J, Ying W Q, Wang Y, Nie Y H, Zhang C C, Li S L, Cheng L P, Wang Q F, Wu Y, Huang P Y, Sun Q, Shi L Y, Yang H. Homology-mediated end joining-based targeted integration using CRISPR/Cas9. Cell Research, 2017, 27(6): 801-814

53. Suzuki K, Tsunekawa Y, Hernandez-Benitez R, Wu J, Zhu J, Kim E J, Hatanaka F, Yamamoto M, Araoka T, Li Z, Kurita M, Hishida T, Li M, Aizawa E, Guo S C, Chen S, Goebl A, Soligalla R D, Qu J, Jiang T S, Fu X, Jafari M, Esteban C R, Berggren W T, Lajara J, Nuñez-Delicado E, Guillen P, Campistol J M, Matsuzaki F, Liu G H, Magistretti P, Zhang K, Callaway E M, Zhang K, Belmonte J C I. In vivo genome editing via CRISPR/Cas9 mediated homologyindependent targeted integration. Nature, 2016, 540(7631): 144149

54. Liu X, Wang Y Z, Gao Y P, Su J M, Zhang J C, Xing X P, Zhou C, Yao K Z, An Q, Zhang Y. H3K9 demethylase KDM4E is an epigenetic regulator for bovine embryonic development and a defective factor for nuclear reprogramming. Development, 2018, 145(4): dev158261

55. Holm P, Shukri N N, Vajta G, Booth P, Bendixen C, Callesen H. Developmental kinetics of the first cell cycles of bovine in vitro produced embryos in relation to their in vitro viability and sex. Theriogenology, 1998, 50(8): 1285-1299

56. Wrenzycki C. In vitro Production of (Farm) Animal Embryos. In: Niemann H, Wrenzycki C, eds. Animal Biotechnology 1: Reproductive Biotechnologies. Cham: Springer, 2018, 269-304

57. Aiken C E M, Swoboda P P L, Skepper J N, Johnson M H. The direct measurement of embryogenic volume and nucleo-cytoplasmic ratio during mouse pre-implantation development. Reproduction, 2004, 128(5): 527-535

58. Willadsen S M, Polge C. Attempts to produce monozygotic quadruplets in cattle by blastomere separation. Veterinary Record, 1981, 108(10): 211-213
59. Tu Z C, Yang W L, Yan S, Yin A, Gao J Q, Liu X D, Zheng Y H, Zheng J Z, Li Z J, Yang S H, Li S H, Guo X Y, Li X J. Promoting Cas 9 degradation reduces mosaic mutations in non-human primate embryos. Scientific Reports, 2017, 7(1): 42081

60. Fisher P J, Hyndman D L, Bixley M J, Oback F C, Popovic L, McGowan L T, Berg M C, Wells D N. Brief communication: potential for genomic selection of bovine embryos. Proceedings of the New Zealand Society of Animal Production, 2012, 72: 156-158

61. Oback F C, Wei J, Popovic L, McGowan L T, Oliver J E, Delaney S R, Wells D N. Blastocyst bisection to multiply biopsied and vitrified bovine embryos. Reproduction, Fertility, and Development, 2016, 29(1): 154

62. Evans M J, Kaufman M H. Establishment in culture of pluripotential cells from mouse embryos. Nature, 1981, 292 (5819): 154-156

63. Martin G R. Isolation of a pluripotent cell line from early mouse embryos cultured in medium conditioned by teratocarcinoma stem cells. Proceedings of the National Academy of Sciences of the United States of America, 1981, 78(12): 7634-7638

64. Capecchi M R. Gene targeting in mice: functional analysis of the mammalian genome for the twenty-first century. Nature Reviews. Genetics, 2005, 6(6): 507-512

65. Mulas C, Kalkan T, von Meyenn F, Leitch H G, Nichols J, Smith A. Defined conditions for propagation and manipulation of mouse embryonic stem cells. Development, 2019, 146(6): dev173146

66. Nagy A, Rossant J, Nagy R, Abramow-Newerly W, Roder J C. Derivation of completely cell culture-derived mice from earlypassage embryonic stem cells. Proceedings of the National Academy of Sciences of the United States of America, 1993, 90 (18): 8424-8428

67. Nichols J, Smith A. Naïve and primed pluripotent states. Cell Stem Cell, 2009, 4(6): 487-492

68. Suda Y, Suzuki M, Ikawa Y, Aizawa S. Mouse embryonic stem cells exhibit indefinite proliferative potential. Journal of Cellular Physiology, 1987, 133(1): 197-201

69. Oback B, Huang B. Pluripotent Stem Cells in Livestock, in Stem Cells: From Basic Research to Therapy. In: Calegari F, Waskow C, eds. Stem Cells: From Basic Research to Therapy, Volume 2: Tissue Homeostasis and Regeneration during Adulthood, Applications, Legislation and Ethics. New York: CRC Press/Taylor \& Francis Group, 2014, 305-346

70. Gao X F, Nowak-Imialek M, Chen X, Chen D S, Herrmann D, Ruan D, Chen A C H, Eckersley-Maslin M A E, Ahmad S, Lee Y L, Kobayashi T, Ryan D, Zhong J X, Zhu J C, Wu J, Lan G C, Petkov S, Yang J, Antunes L, Campos L S, Fu B Y, Wang S P, Yong Y, Wang X M, Xue S G, Ge L P, Liu Z H, Huang Y, Nie T, Li P, Wu D H, Pei D Q, Zhang Y, Lu L M, Yang F T, Kimber S J, Reik W, Zou X G, Shang Z C, Lai L X, Surani A, Tam P P L, Ahmed A, Yeung W S B, Teichmann S A, Niemann H, Liu P. Establishment of porcine and human expanded potential stem cells. Nature Cell Biology, 2019, 21(6): 687-699

71. Bogliotti Y S, Wu J, Vilarino M, Okamura D, Soto D A, Zhong C Q, Sakurai M, Sampaio R V, Suzuki K, Izpisua Belmonte J C I, Ross P J. Efficient derivation of stable primed pluripotent embryonic stem cells from bovine blastocysts. Proceedings of the National Academy of Sciences of the United States of America, 
2018, 115(9): 2090-2095

72. Buecker C, Chen H H, Polo J M, Daheron L, Bu L, Barakat T S, Okwieka P, Porter A, Gribnau J, Hochedlinger K, Geijsen N. A murine ESC-like state facilitates transgenesis and homologous recombination in human pluripotent stem cells. Cell Stem Cell, 2010, 6(6): 535-546

73. Hockemeyer D, Soldner F, Beard C, Gao Q, Mitalipova M, DeKelver R C, Katibah G E, Amora R, Boydston E A, Zeitler B, Meng X, Miller J C, Zhang L, Rebar E J, Gregory P D, Urnov F D, Jaenisch R. Highly efficient gene targeting of expressed and silent genes in human ESCs and iPSCs using zinc-finger nucleases. Nature Biotechnology, 2009, 27(9): 851-857

74. Hockemeyer D, Wang H Y, Kiani S, Lai C S, Gao Q, Cassady J P, Cost G J, Zhang L, Santiago Y, Miller J C, Zeitler B, Cherone J M, Meng X D, Hinkley S J, Rebar E J, Gregory P D, Urnov F D, Jaenisch R. Genetic engineering of human pluripotent cells using TALE nucleases. Nature Biotechnology, 2011, 29(8): 731-734

75. Hockemeyer D, Jaenisch R. Induced pluripotent stem cells meet genome editing. Cell Stem Cell, 2016, 18(5): 573-586

76. Heyman Y, Chavatte-Palmer P, LeBourhis D, Camous S, Vignon $\mathrm{X}$, Renard J P. Frequency and occurrence of late-gestation losses from cattle cloned embryos. Biology of Reproduction, 2002, 66(1): 6-13

77. Misica-Turner P M, Oback F C, Eichenlaub M, Wells D N, Oback B. Aggregating embryonic but not somatic nuclear transfer embryos increases cloning efficiency in cattle. Biology of Reproduction, 2007, 76(2): 268-278

78. Oback B. Cloning from stem cells: different lineages, different species, same story. Reproduction, Fertility, and Development, 2009, 21(1): 83-94

79. Eggan K, Rode A, Jentsch I, Samuel C, Hennek T, Tintrup H, Zevnik B, Erwin J, Loring J, Jackson-Grusby L, Speicher M R, Kuehn R, Jaenisch R. Male and female mice derived from the same embryonic stem cell clone by tetraploid embryo complementation. Nature Biotechnology, 2002, 20(5): 455-459

80. Humpherys D, Eggan K, Akutsu H, Hochedlinger K, Rideout W M 3rd, Biniszkiewicz D, Yanagimachi R, Jaenisch R. Epigenetic instability in ES cells and cloned mice. Science, 2001, 293(5527): 95-97

81. Ideta A, Yamashita S, Seki-Soma M, Yamaguchi R, Chiba S, Komaki H, Ito T, Konishi M, Aoyagi Y, Sendai Y. Generation of exogenous germ cells in the ovaries of sterile NANOS3-null beef cattle. Scientific Reports, 2016, 6(1): 24983

82. Tsuda M, Sasaoka Y, Kiso M, Abe K, Haraguchi S, Kobayashi S, Saga Y. Conserved role of nanos proteins in germ cell development. Science, 2003, 301(5637): 1239-1241

83. Ruggiu M, Speed R, Taggart M, McKay S J, Kilanowski F, Saunders P, Dorin J, Cooke H J. The mouse Dazla gene encodes a cytoplasmic protein essential for gametogenesis. Nature, 1997, 389 (6646): 73-77

84. Park K E, Kaucher A V, Powell A, Waqas M S, Sandmaier S E S, Oatley M J, Park C H, Tibary A, Donovan D M, Blomberg L A, Lillico S G, Whitelaw C B A, Mileham A, Telugu B P, Oatley J M.
Generation of germline ablated male pigs by CRISPR/Cas9 editing of the NANOS2 gene. Scientific Reports, 2017, 7(1): 40176

85. McLean Z, Appleby S J, Wei J W, Snell R G, Oback B. Testes of DAZL null sheep lack spermatogonia and maintain normal somatic cells. bioRxiv, 2019 [Preprint] doi: 10.1101/848036

86. Giassetti M I, Ciccarelli M, Oatley J M. Spermatogonial stem cell transplantation: insights and outlook for domestic animals. Annual Review of Animal Biosciences, 2019, 7(1): 385-401

87. Fehilly C B, Willadsen S M, Tucker E M. Experimental chimaerism in sheep. Journal of Reproduction and Fertility, 1984, 70(1): 347-351

88. Picard L, Chartrain I, King W A, Betteridge K J. Production of chimaeric bovine embryos and calves by aggregation of inner cell masses with morulae. Molecular Reproduction and Development, 1990, 27(4): 295-304

89. Brem G, Tenhumberg H, Krausslich H. Chimerism in cattle through microsurgical aggregation of morulae. Theriogenology, 1984, 22(5): 609-613

90. Nakano K, Watanabe M, Matsunari H, Matsuda T, Honda K, Maehara M, Kanai T, Hayashida G, Kobayashi M, Kuramoto M, Arai Y, Umeyama K, Fujishiro S H, Mizukami Y, Nagaya M, Hanazono Y, Nagashima H. Generating porcine chimeras using inner cell mass cells and parthenogenetic preimplantation embryos. PLoS One, 2013, 8(4): e61900

91. Onishi A, Takeda K, Komatsu M, Akita T, Kojima T. Production of chimeric pigs and the analysis of chimerism using mitochondrial deoxyribonucleic acid as a cell marker. Biology of Reproduction, 1994, 51(6): 1069-1075

92. Hayashi K. In vitro reconstitution of germ cell development. Biology of Reproduction, 2019, 101(3): 567-578

93. Haley C S, Visscher P M. Strategies to utilize marker-quantitative trait loci associations. Journal of Dairy Science, 1998, 81 (Supplement 2): 85-97

94. Friedrichs S, Takasu Y, Kearns P, Dagallier B, Oshima R, Schofield J, Moreddu C. An overview of regulatory approaches to genome editing in agriculture. Biotechnology Research and Innovation, 2019, 3(2): 208-220

95. Ledford H. CRISPR conundrum: Strict European court ruling leaves food-testing labs without a plan. Nature, 2019, 572(7767): 15

96. Food and Drug Administration (FDA). Guidance for industry \#187: regulation of intentionally altered genomic DNA in animals. Available at the FDA website on September 21, 2019

97. Lema M A. Regulatory aspects of gene editing in Argentina. Transgenic Research, 2019, 28(Supplement 2): 147-150

98. Thygesen P. Clarifying the regulation of genome editing in Australia: situation for genetically modified organisms. Transgenic Research, 2019, 28(Supplement 2): 151-159

99. Ellens K W, Levac D, Pearson C, Savoie A, Strand N, Louter J, Tibelius C. Canadian regulatory aspects of gene editing technologies. Transgenic Research, 2019, 28(2): 165-168

100. Wells D N, Oback B, Laible G. Cloning livestock: a return to embryonic cells. Trends in Biotechnology, 2003, 21(10): 428-432 